\title{
Beyond ADHD and narcolepsy: psychostimulants in general psychiatry
}

\author{
Bradley Ng \& Angela O’Brien
}

\begin{abstract}
SUMMARY
Psychostimulants (dexamphetamine, methylphenidate, modafinil) reduce fatigue, promote alertness and wakefulness, and have possible mood-enhancing properties. In modern psychiatric practice, their use has been limited to attentiondeficit hyperactivity disorder and sleep disorders such as narcolepsy. Despite this, research has continued into psychostimulant use in general psychiatry, especially in the treatment of depression and fatigue. This article reviews the recent literature regarding psychostimulant use in general and consultation-liaison psychiatry. Although psychostimulants continue to attract clinical research, there is currently not enough evidence to recommend their routine use for general psychiatric conditions.
\end{abstract}

\section{DECLARATION OF INTEREST}

None.

Psychostimulants are a broad class of drugs that reduce fatigue, promote alertness and wakefulness, and have possible mood-enhancing properties (Orr 2007). Healthcare professionals have used amphetamine and methylphenidate since the early 1930s to treat people diagnosed with various conditions, including depression, fatigue, neurasthenia, weight loss and hypersomnolence. In the 1960s, multiple randomised controlled trials (RCTs) questioned the efficacy of psychostimulants in major depression against placebo, tricyclic antidepressants and monoamine oxidase inhibitors. Concerns were also raised about misuse and tolerance, leading to their decreasing use in general psychiatry (Chiarello 1987).

In modern psychiatric practice, psychostimulants have a limited but important and well-identified role. The older agents (dexamphetamine, methylphenidate and pemoline) are used to treat attentiondeficit hyperactivity disorder (ADHD) in both children and adults. A newer psychostimulant, modafinil, has US Food and Drug Administration and UK Medicines and Healthcare products Regulatory Agency (MHRA) approval for reducing

sleepiness in obstructive sleep apnoea, narcolepsy and shift-work sleep disorder.

Research has continued into the use of psychostimulants to treat a range of affective, cognitive and somatic symptoms. This article will review the literature for psychostimulant use in adults for a variety of medical, neurological and psychiatric conditions that are relevant to modern psychiatric practice (see Box 1 for search method).

\section{Pharmacology}

\section{Amphetamine}

Amphetamine is an organic molecule that is an indirectly acting sympathomimetic, with its d-isomer, dexamphetamine, being three to four times more active than the l-isomer. Its mechanism of action is thought to be the increasing of noradrenaline and dopamine by blocking their reuptake and facilitating their release at central presynaptic neurons. It is also a weak monoamine oxidase inhibitor (Chiarello 1987; Masand 1996). The stimulation of the dopaminergic mesocorticolimbic and nigrostriatal pathways may be responsible for the motor and behavioural effects (Homsi 2000; Medsafe 2007).

\section{Dexamphetamine}

Dexamphetamine is rapidly absorbed and readily crosses the blood-brain barrier. Its central effects include anorexia, vasoconstriction and hyperthermia. Peripherally, it can elevate diastolic and systolic

B0X 1 Search method for this review

We undertook a Medline search for articles published in English between January 1987 and October 2007 on psychostimulant use (key words: psychostimulants, dexamphetamine, amphetamine, methylphenidate, modafinil) in the treatment of symptoms and disorders of interest to psychiatrists (key words: depression, cognition, fatigue, apathy, schizophrenia, dementia).

\section{ARTICLE}

Bradley $\mathbf{N g}$ is a consultant psychiatrist with the Gold Coast Mental Health Service, Queensland, Australia, and an assistant professor at the Faculty of Health Sciences and Medicine, Bond University, Queensland, Australia. He was a senior registrar in old age psychiatry in Auckland, New Zealand, and an honorary lecturer at the University of Auckland. His interests include consultation-liaison psychiatry and short-term psychotherapies. Angela O'Brien is a consultant psychiatrist at Waitemata District Health Board, Auckland, New Zealand. She completed her basic psychiatry training at the Royal Free Hospital, London, and was a senior registrar in old age psychiatry in Auckland. Her practice focuses on both consultation-liaison and inpatient rehabilitation psychiatry for patients under the care of Mental Health Services for Older People. Correspondence Bradley Ng, Older Persons Mental Health, Robina Hospital, Bayberry Lane, Robina, Queensland 4226, Australia. Email: bkwng@hotmail.com. 
blood pressure and has a weak bronchodilatory action (Homsi 2000). In healthy people, it increases wakefulness, alertness, motivation and initiative, and elevates mood (Masand 1996). Given its enhanced potency, the d-isomer is most commonly used in clinical practice and nearly all of the studies of psychostimulant use for depression have used dexamphetamine.

\section{Methylphenidate}

Methylphenidate is a piperidine derivative and its exact mechanism of action is unknown. It binds to dopamine transporters on the presynaptic membrane, particularly in the striatum, resulting in an increase in extrasynaptic dopamine levels. It may also regulate dopamine homeostasis at the nerve terminal and has some effect on noradrenaline reuptake and binds weakly to the serotonin transporter. Unlike dexamphetamine, it does not facilitate dopamine release at the nerve terminal (Challman 2000). Clinically, methylphenidate is considered a milder psychostimulant than the amphetamine derivatives.

\section{Modafinil}

Modafinil is a mild psychostimulant derived from benzhydrylsulfinylacetamide and is structurally unrelated to methylphenidate and amphetamine. Its mechanism of action is unknown and evidence suggests that it is dissimilar to older psychostimulants. Animal studies indicate that modafinil's primary activity may be related to a decrease in gamma-aminobutyric acid-mediated neurotransmission. Other in vitro studies have demonstrated that modafinil also interacts with other neurotransmitter pathways such as glutamine in the hypothalamus, serotonin in the prefrontal cortex and hypothalamus, dopamine in the prefrontal cortex and noradrenaline in the ventrolateral preoptic

TABLE 1 Pharmacokinetics of the major psychostimulants

\begin{tabular}{|llll|}
\hline & Dexamphetamine & Methylphenidate & Modafinil \\
\hline First-pass metabolism & Low & Extensive & Minimal \\
\hline Plasma protein binding & Nil & Low & $60 \%$ \\
\hline Peak plasma concentration, h & $2-4$ & $1-3$ & $2-3$ \\
\hline Average half-life, h & $10-12$ (adults) & $2-7$ (adults) & 17 \\
\hline Active metabolites & None & Minimal & None \\
\hline Elimination & Renal & Renal & $\begin{array}{l}\text { Hepatic } \\
\text { metabolism, } \\
\text { then renal }\end{array}$ \\
\hline CYP450 interactions & & & $\begin{array}{l}\text { Inhibits 2C19 } \\
\text { Induces 3A4/5, } \\
\text { 1A2, 2B6 }\end{array}$ \\
\hline
\end{tabular}

Source: Keating (2005), Homsi (2000). nucleus. It also increases neuronal activity selectively in the hypothalamus, activating tuberomammillary nucleus neurons, which release histamine and neurons in the perifornical area, in turn releasing orexin/hypocretin. These cell groups are associated with the control of wakefulness (Keating 2005). Modafinil is proposed to have less potential for misuse compared with methylphenidate (Jasinski 2000).

Nearly all of the studies discussed below have used standard-release formulations of the psychostimulants. Table 1 shows the pharmacokinetics of dexamphetamine, methylphenidate and modafinil.

\section{Major depression}

\section{Monotherapy}

There have been several reviews of psychostimulants as monotherapy for primary unipolar depression (Satel 1989; Orr 2007). Most RCTs have compared the older psychostimulants with placebo or antidepressants such as imipramine and phenelzine. There have been no RCTs with modafinil. Most of the trials are now more than 20 years old and have many methodological shortcomings, such as varying diagnostic criteria and the use of unvalidated rating scales or global impression alone (Orr 2007). Most studies in non-elderly adults (under 65 years of age) were negative and, in the positive studies, stimulants were only modestly beneficial to certain subgroups compared with placebo.

In contrast to this there have been some early, but less than methodologically robust, positive trials of monotherapy psychostimulant use to treat depression in elderly people (Jacobson 1958; Kaplitz 1975). The most recent RCT (Wallace 1995) was positive but involved only 16 medically ill patients with depression in a very short trial.

Overall, this treatment option has a poor evidence base. It may have some utility for elderly people or for treating depression in the medically unwell; this will be discussed below.

\section{Adjunctive treatment}

There are several positive open trials, audits and case reports regarding the use of psychostimulants to augment standard antidepressant treatment, including tricyclic antidepressants (Gwirtsman 1994), monoamine oxidase inhibitors (Feinberg 2004), selective serotonin reuptake inhibitors (Ninan 2004), venlafaxine (Masand 1998) and mirtazapine (Schillerstrom 2002).

The two negative RCTs using methylphenidate as an augmentation agent compared with placebo in adults with major depression showed no statistically significant improvement or accelerated response 
(Postolache 1999; Patkar 2006). Patkar and colleagues (2006) noted that in 60 out-patients with treatment-resistant depression, more participants responded to extended-release methylphenidate compared with placebo (40\% v. 23.3\%). However, this difference was not statistically significant. There were no differences in the rate of adverse events or the number of participants who dropped out of the trial.

Lavretsky and colleagues (2006) conducted an RCT involving 16 elderly participants with major depression, comparing methylphenidate with placebo augmentation, commenced simultaneously with citalopram. There was an accelerated antidepressant response by week 3 and a greater reduction in depression scores in the participants on the combination treatment. However, side-effects and intolerability caused a number of participants to drop out of the trial.

There have been two well-designed RCTs comparing modafinil with placebo as an augmentation agent to antidepressants in the treatment of nonelderly people with major depression. Participants in these trials had experienced a partial response to an antidepressant, usually a selective serotonin reuptake inhibitor, and had significant and persistent fatigue or sleepiness. At the end of both trials, there was no significant difference in depression scores between the two groups. A 6-week trial (DeBattista 2003) involving 168 patients reported a significant improvement compared with placebo for sleepiness at week 1 and fatigue at week 2 , but not at the end of the trial. An 8-week trial (Fava 2005) including 311 patients reported a non-significant trend towards greater mean reductions on depressive scores at the end of the trial for the modafinil group. Participants on modafinil experienced more adverse events, notably nausea and feeling jittery.

The evidence base for using psychostimulants as augmentation agents for major unipolar depression is limited (Orr 2007). It remains to be seen whether psychostimulants may benefit a subgroup of people with depression who also have significant fatigue or sleep problems. A systematic review found inadequate evidence for modafinil as an augmentation agent in the treatment of fatigue in individuals with major depression who have had a partial response to antidepressants (Lam 2007).

\section{Bipolar depression}

Bipolar depression remains a major challenge in general adult psychiatry. The older psychostimulants have not been recommended owing to a lack of proven efficacy, mood-switching potential, tolerance and misuse. Modafinil has been used in open trials to treat bipolar depression, either as monotherapy or as an adjunct to standard antidepressant treatment (Carlson 2004). Benefits over the older psychostimulants include less peripheral activation, decreased anxiogenic properties and lower misuse potential (Jasinski 2000).

A subsequent RCT compared 6 weeks of adjunctive modafinil with placebo in 85 people with bipolar depression who were on a mood stabiliser, with or without concomitant antidepressant treatment (Frye 2007). The modafinil group had significantly higher remission (39\% v. 18\%) and response (44\% v. 23\%) rates, with significant improvements noted by week 2 . There were no differences in treatmentemergent mania or hypomania during the study period between the two groups, and being on concomitant antidepressant treatment was not a factor in response or remission. Modafinil appeared to be well tolerated.

Although still preliminary and needing replication, this trial was well designed and provided evidence of a possible novel treatment option for bipolar depression. Modafinil's advantages included its relative safety, lack of mood-switching effect and relatively fast antidepressant response. Questions remain about the ideal dosage, any specific effects on energy and sleepiness, and the actual mechanism of action.

\section{Depression in medical illness}

An extensive literature exists regarding the use of dexamphetamine and methylphenidate as monotherapy for affective disorders in a wide variety of medically or surgically unwell patients. The case series and large retrospective audits summarised by Masand $\&$ Tesar (1996) reported clinical response rates between 48 and 70\%. In individual patients, the results were sometimes dramatic, with an antidepressant response within 2-3 days and with minimal side-effects. The recorded benefits include patients being discharged earlier than anticipated, engagement in rehabilitation, an absence of major side-effects and minimal drug interactions. The incidence of hypomania was rare (Masand 1995). Reported disadvantages include a lack of a solid evidence base, potential medication interactions and stigma surrounding psychostimulants (Frierson 1991).

There have been few RCTs involving medically ill patients. Landman and colleagues (1958) performed a crossover trial with 112 medically ill patients with mild depression, comparing methylphenidate with placebo. Methylphenidate was clearly superior and clearly differentiated from placebo. In another of these trials, Wallace and colleagues (1995) conducted a small double-blind crossover trial in 16 medically ill elderly patients. Again, 
methylphenidate demonstrated a significant benefit over placebo. However, the trial's small numbers and high mortality rate make the results difficult to interpret. Modafinil has not undergone RCTs in medically ill people, although there have been positive case reports of its use (Schwartz 2002).

Both the two trials and the retrospective audits above demonstrate a significant short-term antidepressant effect with psychostimulants in medically ill people, but the long-term benefits and risks associated with extended treatment are still unknown. Although depressive symptoms have been the main indication for psychostimulant prescription in these cases, the diagnosis has not necessarily always been major depression or its equivalent.

\section{Cancer and palliative care}

Psychostimulants, particularly methylphenidate, have been studied for treating depression associated with cancer in a wide variety of populations, from ambulatory to palliative care patients. Retrospective audits and open studies report that between 27 and $78 \%$ of patients experience some improvement in depressive symptoms on a psychostimulant (Rozans 2002). None of the studies in depression has been an RCT, which may be difficult to undertake in this population. Furthermore, there may have been a nonspecific euphoriant effect. Despite the lack of robust evidence, many authorities have recommended the use of psychostimulants, especially in depression in palliative care, when a fast response is required in a limited amount of time (Block 2000).

Fatigue is also a significant symptom in this patient population and may be due to the cancer itself or the related treatments. Open studies were initially encouraging but an RCT of 31 patients comparing methylphenidate with placebo found no significant difference between the two groups at 1 and 4 weeks of open-label extension (Bruera 2006).

One open study and one RCT found significant benefits in cognition for people with cancer treated with psychostimulants (Rozans 2002). Cognitive impairment in cancer may be due to the process of the disease, metabolic abnormalities, opiate use or the side-effects of radiation or chemotherapy. Finally, methylphenidate has been of benefit for hypoactive delirium in palliative care settings (Keen 2004).

\section{Stroke}

\section{Post-stroke depression}

There have been positive retrospective audits and open trials of psychostimulants, predominantly methylphenidate, in post-stroke depression (Kraus 1995). Partial or full remission was reported in 40 to $80 \%$ of patients, with doses ranging from 5 to $40 \mathrm{mg}$ daily, over $2-4$ weeks. Stimulants were well tolerated and treatment response usually occurred within 1-3 days.

Lazarus and colleagues (1994) noted no difference in the degree of response to methylphenidate compared with nortriptyline. However, the average speed of response was significantly different (2.4 days for methylphenidate compared with 27 days for nortriptyline). Grade and colleagues (1998) noted a significant improvement with 3 weeks of methylphenidate in Hamilton Rating Scale for Depression scores. In contrast, a Cochrane review (Martinsson 2007) found no benefit for dexamphetamine for depression in the context of post-stroke rehabilitation.

An important consideration in using stimulants in the post-stroke period is the potential for an elevation in blood pressure. In the absence of robust post-stroke data, it is worth considering a braininjury study (Alban 2004) that reported minimal adverse effects of methylphenidate on pulse rate and blood pressure (which increased by $7 \mathrm{bpm}$ and $2.5 \mathrm{mmHg}$ respectively).

\section{Stroke rehabilitation}

Martinsson and colleagues' (2007) Cochrane review on the use of dexamphetamine given over 1-5 weeks in the post-stroke period, usually in combination with physical or cognitive training, showed benefits of treatment on motor and language functioning, but not on neurological functioning, performance of activities of daily living or depression. A nonsignificant increase in mortality was observed with dexamphetamine. The authors concluded that dexamphetamine could not be recommended routinely in stroke rehabilitation.

This review and an RCT (Sonde 2007) suggest that psychostimulants should not be used routinely in post-stroke rehabilitation. However, they might be tried in individual patients to target symptoms such as depression, fatigue and sleepiness, if other treatments have been ineffectual or are contraindicated.

\section{Traumatic brain injury}

Psychostimulants have a potential therapeutic role in ameliorating the neuropsychiatric sequelae of traumatic brain injury. Most studies have used methylphenidate at doses of $0.3 \mathrm{mg} / \mathrm{kg}$ given twice daily to patients with moderate to severe traumatic brain injury (Whyte 2002).

Although there is a dearth of RCTs with sufficient power to draw any definitive conclusions or to allow psychostimulants to be recommended as routine therapy, this group of agents may be worth considering for impairing symptoms that have failed to respond to conventional treatment. 


\section{Acute and subacute recovery}

A meta-analysis of controlled single-subject trials involving individuals included at a mean of 7.5 days after traumatic brain injury showed no effect of methylphenidate on command-following or yes/ no communication abilities (Martin 2007). Kaelin and colleagues (1996) reported an improvement in a number of attentional domains in a prospective study of 11 adults who received 10 days of methylphenidate, at a mean duration of 20 days after traumatic brain injury.

In an RCT of methylphenidate over 30 days in the subacute phase of traumatic brain injury, significant differences were seen on measures of attention, motor performance and disability after treatment, but this was not the case at 90 days (Plenger 1996). Lee and colleagues (2005) described a 4-week RCT involving 30 patients who had experienced a major depressive episode, comparing methylphenidate with sertraline or placebo in treatment that began a mean of 35 days after traumatic brain injury. The study showed significant improvements in postconcussional symptoms, daytime wakefulness and reaction time with methylphenidate compared with placebo. Sertraline led to impaired performance in these domains.

\section{Cognition}

The rationale for the use of psychostimulants in cognitive rehabilitation after traumatic brain injury is derived from their use in $\mathrm{ADHD}$, where methylphenidate has been shown to reduce hyperactivity, increase on-task behaviour and enhance working memory (Whyte 1997).

A number of small crossover RCTs of methylphenidate in adults with traumatic brain injury have been reported. Treatment duration varied between 10 days and 6 weeks, with a mean time since injury of 1.4-3.9 years. Two of the studies were negative (Gualtieri 1988; Speech 1993). However, Whyte and colleagues $(1997,2004)$ reported significant improvements in domains related to cognitive speed and response rate, but not in attentional domains or motor speed. They concluded that methylphenidate improved 'initial performance', but may not enhance patients' ability to maintain performance over time.

One parallel-group RCT has been conducted, with cognitive functioning as a secondary outcome measure. Mooney \& Haas (1993) studied the effects of methylphenidate over 6 weeks at a mean of 2 years after traumatic brain injury. No benefits were noted regarding attention or memory, but participants with higher pre-treatment anger scores did show a significant improvement on a verbal learning task.

\section{Depression}

Lee and colleagues (2005) noted significant improvements in mood with methylphenidate over 4 weeks; sertraline had a similar efficacy but a higher total number of adverse events. Thus, methylphenidate may be both beneficial and tolerable in the early stages of treatment for a depressive episode after traumatic brain injury, but it is unclear whether these results would be sustained over a longer period of time.

\section{Behaviour}

Mooney \& Haas (1993) reported significant improvements in anger control and psychopathology with 6 weeks of methylphenidate treatment. Patients with higher pre-treatment anger scores showed the greatest benefit. Speech and colleagues (1993) found no effects of methylphenidate for differences in 'social personality' functioning on the Katz Adjustment Scale, despite the majority of patients and observers reporting improvements during the drug condition.

\section{Psychiatric symptoms in other neurological disorders}

\section{Depression}

Psychostimulants have been used to treat depression in people with neurological disorders, including dementia, Parkinson's disease, epilepsy, neuropathy, normal pressure hydrocephalus and multiple sclerosis. However, data have been restricted to case series or large retrospective audits involving medically ill patients.

\section{Apathy}

Apathy is a common and disabling symptom seen in a range of neuropsychiatric and mental disorders. A number of case reports have noted the successful use of methylphenidate, dexamphetamine and modafinil for treating apathy in a range of neurological conditions (Padala 2007).

Early studies suggested that psychostimulants may be useful for apathy in people with varying degrees of cognitive impairment or dementia, with or without depression, but it was unclear whether the improvements were due to changes in affect, motivation or cognition, or a combination of the three (Moore 1981). Galynker and colleagues (1997) prospectively involved 17 individuals with dementia and found a significant improvement in negative symptoms with methylphenidate treatment.

Kaplitz (1975) remains the only RCT of psychostimulants for apathy. It reported a positive effect of methylphenidate v. placebo in 'withdrawn, apathetic geriatric patients'. 
In light of the above evidence, it might be worth considering prescribing a psychostimulant for treatment-resistant apathy, regardless of aetiology, particularly in older adults.

\section{Chronic fatigue syndrome}

Psychostimulants have been trialled in people with chronic fatigue syndrome. Olson and colleagues (2003) conducted a 6-week RCT in 20 patients, comparing dexamphetamine (up to $15 \mathrm{mg}$ twice a day) with placebo. The medication group showed a statistically significant reduction on the Fatigue Severity Scale. Other outcome measures, including quality-of-life patient-determined outcomes, were negative. The authors concluded that dexamphetamine may be useful in managing chronic fatigue syndrome.

There have been two crossover trials since: one with modafinil, which was negative (Randall 2005), and one with methylphenidate, which was modestly positive (Blockmans 2006). These studies suggest that psychostimulants do not have a routine role in chronic fatigue syndrome.

\section{HIV/AIDS}

Evidence from RCTs provides support for the efficacy of psychostimulants in the treatment of HIV/AIDS-associated depression. Significant improvements in mood, energy levels and quality of life were seen in patients with depressive-spectrum disorders and fatigue treated with 2 weeks of dexamphetamine (Wagner 2000). Fernandez and colleagues (1995) found no difference over 6 weeks between methylphenidate and desipramine in HIVpositive individuals with a major depressive episode. Both groups showed significant improvements in mood, although methylphenidate did not have a more rapid onset of efficacy than desipramine.

Subjective fatigue occurs in up to $50 \%$ of patients during the course of HIV/AIDS (Breitbart 2001). There is reasonable RCT evidence to support the use of methylphenidate, pemoline and modafinil for HIV-related fatigue, with associated improvements seen in mood, distress levels and quality of life (Breitbart 2001; Rabkin 2004).

Small prospective and crossover studies have reported positive effects of methylphenidate, dexamphetamine and modafinil on measures of cognition, including memory, processing speed and executive functioning in HIV/AIDS (Rabkin 2004). However, negative trials also exist (Hinkin 2001) and improvement has been observed in placebo groups, suggesting the possibility of carry-over and/ or practice effects.

Positive effects have not yet been found in RCTs for methylphenidate (Fernandez 1995) or dexamphetamine (Wagner 2000) for cognitive functioning in HIV. Therefore, psychostimulants cannot currently be recommended as a treatment for this symptom cluster.

\section{Other areas of psychiatry}

The successful use of modafinil and methylphenidate to treat sedation caused by psychotropic drugs has been noted in case reports and uncontrolled studies in relation to sodium valproate (Berigan 2004), antipsychotics, including clozapine (Miller 1996), and selective serotonin reuptake inhibitors (Schwartz 2004). The possibility of adverse effects must be considered whenever using psychostimulants in people with psychotic disorders, with case reports noting the exacerbation of psychotic symptoms with modafinil (Narendran 2002) and movement disorder symptoms with methylphenidate (Miller 1996).

Rosenthal \& Bryant (2004) described benefits of modafinil on clinical presentation, global functioning and fatigue in a prospective study of individuals with schizophrenia and schizoaffective disorder. However, an RCT of modafinil for the alleviation of fatigue and cognitive deficits in a similar population found no benefit of modafinil over placebo (Sevy 2005). One participant withdrew from each of these studies because of worsening psychosis.

One RCT reported benefits of modafinil on abstinence rates in cocaine dependence (Dackis 2005), whereas another (Grabowski 1997) found no benefits with methylphenidate.

\section{Adverse effects}

Common side-effects of older psychostimulants include insomnia, agitation, anxiety and confusion (Orr 2007). Although such side-effects are generally considered uncommon and short-lived when treatment is continued, one must be cautious when interpreting these results and drawing conclusions from the limited evidence available, which is usually related to children and adolescents treated for ADHD. The potential for misuse must always be considered, but many authors who have prescribed psychostimulants regularly for medical purposes have not noted misuse to be a significant problem (Masand 1996; Olson 2003).

The cardiovascular changes associated with older psychostimulants have been highlighted and concerns about sudden death led the US Food and Drug Administration to recommend that psychostimulant drugs used to treat ADHD include a black-box warning (Nissen 2006). It was speculated that older psychostimulants could increase heart rate and blood pressure, leading to chronic heart failure and arrhythmias with 
long-term use. The warning is primarily directed at the use of psychostimulants to treat children and adolescents with ADHD. It is not clear how psychostimulant use would affect adults being treated for conditions other than ADHD. Heart rate and blood pressure changes associated with psychostimulant use are modest but measurable (Alban 2004). Furthermore, psychostimulant use in adults may be only short term and at different doses than for ADHD treatment. The UK's MHRA has recently issued drug safety advice regarding methylphenidate and potential cardiovascular and cerebrovascular risks (Medicines and Healthcare products Regulatory Agency 2009).

Side-effects of modafinil include headache, nausea and nervousness, with only the first symptom reaching significantly greater frequency than placebo in clinical trials. Most adverse effects are mild to moderate and large overdoses of modafinil alone have had no fatal outcomes (Keating 2005). Despite a generally benign safety profile, the MHRA has recently issued concerns about modafinil and the risk of serious skin reactions and possible exacerbation of psychiatric symptoms (Medicines and Healthcare products Regulatory Agency 2008).

There has always been a theoretical risk of inducing mania or hypomania when using psychostimulants and case reports have been published, but these are considered rare in the adult population (Masand 1995; Orr 2007). In a trial for bipolar depression, modafinil did not have higher rates of mania or hypomania compared with placebo (Frye 2007).

\section{Conclusions}

Currently, routine use of psychostimulants cannot be recommended for any of the conditions described above. Most trials have been very short and maintenance and follow-up data are non-existent. The evidence gathered to date would suggest, however, that these agents should be considered for specific symptoms such as depression, apathy, fatigue and sleepiness in a range of disorders. In clinical practice, the use of psychostimulants would be most appropriate where traditional agents have been ineffectual or contraindicated, a rapid response may be life-saving or lead to a greatly improved outcome or quality of life, and adverse events can be carefully monitored and managed.

\section{References}

Alban JP, Hopson MM, Ly V, et al (2004) Effect of methylphenidate on vital signs and adverse effects in adults with traumatic brain injury. American Journal of Physical Medicine and Rehabilitation; 83: 131-7.

Berigan T (2004) Modafinil treatment of excessive sedation associated with divalproex sodium. Canadian Journal of Psychiatry; 49: 72-3.

Block SD (2000) Assessing and managing depression in the terminally ill patient. Annals of Internal Medicine; 132: 209-18.
Blockmans D, Persoons P, Van Houdenhove B, et al (2006) Does methylphenidate reduce the symptoms of chronic fatigue syndrome? American Journal of Medicine; 119: 167.e23-30.

Breitbart W, Rosenfeld B, Kaim M, et al (2001) A randomized, doubleblind, placebo-controlled trial of psychostimulants for the treatment of fatigue in ambulatory patients with human immunodeficiency virus disease. Archives of Internal Medicine; 161: 411-20.

Bruera E, Valero V, Driver L, et al (2006) Patient-controlled methylphenidate for cancer fatigue. A double-blind, randomized, placebo-controlled trial. Journal of Clinical Oncology, 24: 2073-8.

Carlson PJ, Merlock MC, Suppes T (2004) Adjunctive stimulant use in patients with bipolar disorder. Treatment of residual depression and sedation. Bipolar Disorders; 6: 416-20.

Challman TD, Lipsky JJ (2000) Methylphenidate. Its pharmacology and uses. Mayo Clinic Proceedings; 75: 711-21.

Chiarello RJ, Cole JO (1987) The use of psychostimulants in general psychiatry. A reconsideration. Archives of General Psychiatry, 44: 286-95.

Dackis CA, Kampman KM, Lynch KG, et al (2005) A double-blind, placebo-controlled trial of modafinil for cocaine dependence. Neuropsychopharmacology, 30: 205-11.

DeBattista C, Doghramji K, Menza MA, et al (2003) Adjunct modafinil for the short-term treatment of fatigue and sleepiness in patients with major depressive disorder. A preliminary double-blind, placebo-controlled study. Journal of Clinical Psychiatry, 64: 1057-64.

Fava M, Thase ME, DeBattista C (2005) A multicenter, placebo-controlled study of modafinil augmentation in partial responders to selective serotonin reuptake inhibitors with persistent fatigue and sleepiness. Journal of Clinical Psychiatry, 66: 85-93.

Feinberg SS (2004) Combining stimulants with monoamine oxidase inhibitors. A review of uses and one possible additional indication. Journal of Clinical Psychiatry, 65: 1520-4.

Fernandez F, Levy JK, Samley HR, et al (1995) Effects of methylphenidate in HIV-related depression. A comparative trial with desipramine. International Journal of Psychiatry in Medicine; 25: 53-67.

Frierson RL, Wey JJ, Tabler JB (1991) Psychostimulants for depression in the medically ill. American Family Physician; 43: 163-70.

Frye MA, Grunze H, Suppes T, et al (2007) A placebo-controlled evaluation of adjunctive modafinil in the treatment of bipolar depression. American Journal of Psychiatry, 164: 1242-9.

Galynker I, leronimo C, Miner C, et al (1997) Methylphenidate treatment of negative symptoms in patients with dementia. Journal of Neuropsychiatry and Clinical Neurosciences; 9: 231-9.

Grabowski J, Roache JD, Schmitz JM, et al (1997) Replacement medication for cocaine dependence. Methylphenidate. Journal of Clinical Psychopharmacology, 17: 485-8.

Grade C, Redford B, Chrostowski J, et al (1998) Methylphenidate in early post-stroke recovery. A double-blind, placebo-controlled study. Archives of Physical Medicine and Rehabilitation; 79: 1047-50.

Gualtieri CT, Evans RW (1988) Stimulant treatment for the neurobehavioral sequelae of traumatic brain injury. Brain Injury, 2: 273-90.

Gwirtsman HE, Szuba MP, Toren L, et al (1994) The antidepressant response to tricyclics in major depressives is accelerated with adjunctive use of methylphenidate. Psychopharmacology Bulletin; 30: 157-64.

Hinkin CH, Castellon SA, Hardy DJ, et al (2001) Methylphenidate improves HIV-1-associated cognitive slowing. Journal of Neuropsychiatry and Clinical Neurosciences; 13: 248-54.

Homsi J, Walsh D, Nelson KA (2000) Psychostimulants in supportive care. Support Care in Cancer, 8: 385-97.

Jacobson A (1958) The use of Ritalin in psychotherapy of depressions of the aged. Psychiatric Quarterly, 32: 474-83.

Jasinski DR (2000) An evaluation of the abuse potential of modafinil using methylphenidate as a reference. Journal of Psychopharmacology, 14: $53-60$

Kaelin DL, Cifu DX, Matthies B (1996) Methylphenidate effect on attention deficit in the acutely brain-injured adult. Archives of Physical Medicine and Rehabilitation; 77: 6-9. 


\begin{tabular}{|c|c|c|c|c|}
\hline \multicolumn{5}{|c|}{ MCO answers } \\
\hline 1 & 2 & 3 & 4 & 5 \\
\hline a f & af & af & a f & \\
\hline b t & $b f$ & $b f$ & $b t$ & b \\
\hline$c f$ & $\mathrm{ct}$ & $c f$ & $c f$ & C \\
\hline$d f$ & $d f$ & $d f$ & $d f$ & $d$ \\
\hline ef & ef & et & ef & \\
\hline
\end{tabular}

Kaplitz SE (1975) Withdrawn, apathetic geriatric patients responsive to methylphenidate. Journal of the American Geriatrics Society, 23: 271-6.

Keating GM, Raffin MJ (2005) Modafinil. A review of its use in excessive sleepiness associated with obstructive sleep apnoea/hypopnoea syndrome and shift work sleep disorder. CNS Drugs; 19: 785-803.

Keen JC, Brown D (2004) Psychostimulants and delirium in patients receiving palliative care. Palliative \& Supportive Care; 2: 199-202.

Kraus MF (1995) Neuropsychiatric sequelae of stroke and traumatic brain injury. The role of psychostimulants. International Journal of Psychiatry in Medicine; 25: 39-51.

Lam JY, Freeman MK, Cates ME (2007) Modafinil augmentation for residual symptoms of fatigue in patients with a partial response to antidepressants. Annals of Pharmacotherapy, 41: 1005-12.

Landman ME, Peisig R, Perlman M (1958) A practical mood stimulant. Journal of the Medical Society of New Jersey, 55: 55-8.

Lavretsky H, Park S, Siddarth P, et al (2006) Methylphenidate-enhanced antidepressant response to citalopram in the elderly. A double-blind, placebo-controlled pilot trial. American Journal of Geriatric Psychiatry, 14: $181-5$.

Lazarus LW, Moberg PJ, Langsley PR, et al (1994) Methylphenidate and nortriptyline in the treatment of poststroke depression. A retrospective comparison. Archives of Physical Medicine and Rehabilitation; 75: 403-6.

Lee H, Kim S-W, Kim J-M, et al (2005) Comparing effects of methylphenidate, sertraline and placebo on neuropsychiatric sequelae in patients with traumatic brain injury. Human Psychopharmacology, 20: 97-104.

Martin RT, Whyte J (2007) The effects of methylphenidate on command following and yes/no communication in persons with severe disorders of consciousness. A meta-analysis of n-of-1 studies. American Journal of Physical Medicine and Rehabilitation; 86: 613-20.

Martinsson L, Hårdemark H-G, Eksborg S (2007) Amphetamines for improving recovery after stroke. Cochrane Database of Systematic Reviews; issue 1: CD002090

Masand PS, Pickett P, Murray GB (1995) Hypomania precipitated by psychostimulant use in depressed medically ill patients. Psychosomatics; 36: 145-7

Masand PS, Tesar GE (1996) Use of stimulants in the medically ill. Psychiatric Clinics of North America; 19: 516-47.

Masand PS, Anand VS, Tanquary JF (1998) Psychostimulant augmentation of second generation antidepressants. A case series. Depression and Anxiety, 7: 89-91

Medicines and Healthcare products Regulatory Agency (2008) Modafinil: serious skin reactions, hypersensitivity, and psychiatric symptoms. Drug Safety Update; 1(8): 5

Medicines and Healthcare products Regulatory Agency (2009) Methylphenidate: update guidance on safe and effective use in ADHD. Drug Safety Update; 2(8): 2

Medsafe (2007) Dexamphetamine sulphate. Medsafe (http://www. medsafe.govt.nz/profs/datasheet/d/Dexamphetaminesulphatetab.htm).

Miller SC (1996) Methylphenidate for clozapine sedation. American Journal of Psychiatry, 153: 1231-2.

Mooney GF, Haas LJ (1993) Effect of methylphenidate on brain injuryrelated anger. Archives of Physical Medicine and Rehabilitation; 74: 153-60

Moore DP (1981) Methylphenidate in depression and states of apathy. Southern Medical Journal; 74: 347-8.

Narendran R, Young CM, Valenti AM, et al (2002) Is psychosis exacerbated by modafinil? Archives of General Psychiatry, 59: 292-3.

Ninan PT, Hassman HA, Glass SJ, et al (2004) Adjunctive modafinil at initiation of treatment with a selective serotonin reuptake inhibitor enhances the degree and onset of therapeutic effects in patients with major depressive disorder and fatigue. Journal of Clinical Psychiatry, 65: $414-20$

Nissen SE (2006) ADHD drugs and cardiovascular risk. New England Journal of Medicine; 354: 1445-8.
Olson LG, Ambrogetti A, Sutherland DC (2003) A pilot randomized controlled trial of dexamphetamine in patients with chronic fatigue syndrome. Psychosomatics; 44: 38-43.

Orr K, Taylor D (2007) Psychostimulants in the treatment of depression. A review of the evidence. CNS Drugs; 21: 239-57.

Padala PR, Burke WJ, Bhatia SC (2007) Modafinil therapy for apathy in an elderly patient. Annals of Pharmacotherapy, 41: 346-9.

Patkar AA, Masand PS, Pae C-U, et al (2006) A randomized, doubleblind, placebo-controlled trial of augmentation with an extended release formulation of methylphenidate in outpatients with treatment-resistant depression. Journal of Clinical Psychopharmacology, 26: 653-6

Plenger PM, Dixon CE, Castillo RM, et al (1996) Subacute methylphenidate treatment for moderate to moderately severe traumatic brain injury. A preliminary double-blind placebo-controlled study. Archives of Physical Medicine and Rehabilitation; 77: 536-40.

Postolache TT, Rosenthal RN, Hellerstein DJ, et al (1999) Early augmentation of sertraline with methylphenidate. Journal of Clinical Psychiatry, 60: 123-4

Rabkin JG, McElhiney MC, Rabkin R, et al (2004) Modafinil treatment for fatigue in HIV+ patients. A pilot study. Journal of Clinical Psychiatry, 65: $1688-95$

Randall DC, Cafferty FH, Shneerson JM, et al (2005) Chronic treatment with modafinil may not be beneficial in patients with chronic fatigue syndrome. Journal of Psychopharmacology, 19: 647-60.

Rosenthal MH, Bryant SL (2004) Benefits of adjunct modafinil in an open-label, pilot study in patients with schizophrenia. Clinical Neuropharmacology, 27: 38-43.

Rozans M, Dreisbach A, Lertora JJL, et al (2002) Palliative uses of methylphenidate in patients with cancer. A review. Journal of Clinical Oncology, 20: 335-9.

Satel SL, Nelson JC (1989) Stimulants in the treatment of depression. A critical overview. Journal of Clinical Psychiatry, 50: 241-9.

Schillerstrom JE, Seaman JS (2002) Modafinil augmentation of mirtazapine in a failure-to-thrive geriatric inpatient. International Journal of Psychiatry in Medicine; 32: 405-10.

Schwartz TL, Leso L, Beale M, et al (2002) Modafinil in the treatment of depression with severe comorbid medical illness. Psychosomatics; 43. $336-7$.

Schwartz TL, Azhar N, Cole K, et al (2004) An open-label study of adjunctive modafinil in patients with sedation related to serotonergic antidepressant therapy. Journal of Clinical Psychiatry, 65: 1223-7.

Sevy S, Rosenthal MH, Alvir J, et al (2005) Double-blind, placebocontrolled study of modafinil for fatigue and cognition in schizophrenia patients treated with psychotropic medications. Journal of Clinical Psychiatry, 66: 839-43.

Sonde L, Lökk J (2007) Effects of amphetamine and/or L-dopa and physiotherapy after stroke. A blinded randomized study. Acta Neurologica Scandinavica; 115: 55-9.

Speech TJ, Rao SM, Osmon DC, et al (1993) A double-blind controlled study of methylphenidate treatment in closed head injury. Brain Injury, 7. $333-8$

Wagner GJ, Rabkin R (2000) Effects of dextroamphetamine on depression and fatigue in men with HIV. A double-blind, placebo-controlled trial. Journal of Clinical Psychiatry, 61: 436-40.

Wallace AE, Kofoed LL, West AN (1995) Double-blind, placebo-controlled trial of methylphenidate in older, depressed, medically ill patients. American Journal of Psychiatry, 152: 929-31.

Whyte J, Hart T, Schuster K, et al (1997) Effects of methylphenidate on attentional function after traumatic brain injury. A randomized, placebocontrolled trial. American Journal of Physical Medicine and Rehabilitation; 76: $440-450$

Whyte J, Vaccaro M, Grieb-Neff P, et al (2002) Psychostimulant use in the rehabilitation of individuals with traumatic brain injury. Journal of Head Trauma Rehabilitation; 17: 284-99.

Whyte J, Hart T, Vaccaro M, et al (2004) Effects of methylphenidate on attention deficits after traumatic brain injury. A multidimensional, randomized, controlled trial. American Journal of Physical Medicine and Rehabilitation; 83: 401-19. 
MCQs

1 The primary mechanism of action of modafinil:

a releases dopamine at the nerve terminal

b may be through gamma-aminobutyric acidmediated neurotransmission

c targets the dopamine transporter

$\mathrm{d}$ releases serotonin at the nerve terminal

e releases noradrenaline at the nerve terminal.

2 Methylphenidate's half-life in adults is:

a 1-2 hours

b $2-3$ hours

c $2-7$ hours

d $7-10$ hours

e 11-14 hours.
3 In major depression, psychostimulants:

a are effective as a monotherapy antidepressant

b always precipitate mania or hypomania

c have good evidence as an augmentation agent to standard antidepressants

$d$ have good evidence for treating fatigue and sleepiness in depression

e may accelerate an antidepressant response in elderly patients.

4 There is RCT evidence to support the use of psychostimulants in treating:

a apathy in dementia

b fatigue in HIV/AIDS

c cognitive deficits in HIV/AIDS

d cognitive deficits in schizophrenia

e clozapine-related sedation.
5 The most commonly recommended dose of methylphenidate in traumatic brain injuries is:

a $0.15 \mathrm{mg} / \mathrm{kg} / \mathrm{day}$

b $0.15 \mathrm{mg} / \mathrm{kg}$ twice a day

c $0.3 \mathrm{mg} / \mathrm{kg} / \mathrm{day}$

d $0.3 \mathrm{mg} / \mathrm{kg}$ twice a day

e $0.5 \mathrm{mg} / \mathrm{kg} / \mathrm{day}$. 